\title{
High-frequency ultrasound as an adjunct to neural electrophysiology: Evaluation and prognosis of Bell's palsy
}

\author{
SHUO LI ${ }^{1}$, RUI-JUN GUO ${ }^{1}$, XIAO-NING LIANG ${ }^{1}$, YUE WU $^{2}$, WEN CAO $^{1}$, \\ ZHEN-PING ZHANG ${ }^{3}$, WEI ZHAO ${ }^{3}$ and HAI-DONG LIANG ${ }^{4,5}$
}

\begin{abstract}
Departments of ${ }^{1}$ Ultrasound Medicine and ${ }^{2}$ Neurology, Beijing Chaoyang Hospital, Capital Medical University, Beijing 100020; ${ }^{3}$ The First Hospital of Shijiazhuang City, Shijiazhuang, Hebei 050011, P.R. China; ${ }^{4}$ Institute of Medical Engineering and Medical Physics, School of Engineering, Cardiff University, Cardiff CF24 3AA, Wales, UK; ${ }^{5}$ Information Science and Engineering School, Fudan University, Shanghai 200433, P.R. China
\end{abstract}

Received September 9, 2015; Accepted November 18, 2015

DOI: $10.3892 /$ etm.2015.2878

\begin{abstract}
Bell's palsy is a form of temporary facial nerve paralysis that occurs primarily in young adults. Previously, various methods were used to assess outcomes in facial nerve disease. The aim of the present study was to characterize the main branches of the normal and abnormal facial nerve using high-frequency ultrasonography (HFUS). A total of 104 healthy volunteers, 40 patients with acute onset of Bell's palsy and 30 patients who underwent 3 -month routine therapy for Bell's palsy disease were included in the study. The healthy volunteers and patients were selected for HFUS examination and VII nerve conduction. The results showed significant differences in nerve diameter, echogenicity, delitescence and amplitude in different groups. Statistically significant correlations were identified for severity grading in one of the experimental groups during HFUS examinations. In conclusion, HFUS as a complementary technique paired with neural electrophysiology may establish the normal values of facial nerve. Additionally, HFUS was beneficial in the process of evaluation and prognosis of Bell's palsy disease.
\end{abstract}

Correspondence to: Dr Rui-Jun Guo, Department of Ultrasound Medicine, Beijing Chaoyang Hospital, Capital Medical University, 8 Gong-Ti South Road, Beijing 100020, P.R. China

E-mail: guodaoshi@126.com

Dr Zhen-Ping Zhang, The First Hospital of Shijiazhuang City, 36 Fan-Xi Road, Shijiazhuang, Hebei 050011, P.R. China

E-mail: 276264624@qq.com

Abbreviations: HFUS, high-frequency ultrasonography; CSA,crosssectional area; CG, control group; EGA, experimental group A; EGB, experimental group B; NGS2.0, Nerve Grading System 2.0; EMG, electromyography; NC, nerve conduction; MWD, M-wave detection; CMAP, compound muscle action potential; DML, distal motor latency

Key words: Bell's palsy, high-frequency ultrasound, neural electrophysiology, evaluation and prognosis

\section{Introduction}

Bell's palsy is a form of temporary facial nerve paralysis that occurs primarily in young adults. Bell's palsy is a condition usually without aura symptoms and its exact pathophysiology remains to be elucidated (1). The onset of Bell's palsy is sudden and symptoms, including inability to lift the eyebrow or close the eyes (1), usually peak within a few days. The diagnosis, prognosis and curative effect evaluation of Bell's palsy depend primarily on the clinical symptoms. The severity of the disease is classified according to the Facial Nerve Grading System 2.0 (NGS2.0), which classifies the severity of Bell's palsy as grades I-VI (2). Imaging support is required to observe the real facial nerve. Physicians are frequently unable to make a definitive judgment regarding the necessity of treatment continuation for patients in grade I or II. Without access to appropriate and effective methods of evaluation, physicians depend on their clinical experiences to form such a judgment.

Hagino et al (3) demonstrated that for the facial paralysis patients participating in his study, only 15 cases of mastoidectomy and facial nerve canal operation manifested facial nerve edema. However, the degree and the details of nerve edema were not identified.

The prognostic value of magnetic resonance imaging (MRI) remains at the level of a differential diagnosis, such as exclusion of intracranial lesions. MRI results are often referred to as being contrast-enhanced rather than physical nerve swelling (4,5). In Bell's palsy, facial nerve enhancement in the facial canal is the characteristic MR change (6). Bell's paralysis with incomplete recovery in the mastoid segment of the facial nerve was better enhanced than the labyrinthine and geniculate ganglion section in MRI $(7,8)$. High-resolution temporal bone computed tomography (CT) is also able to identify temporal bone fractures that violate the facial nerve canal (9). However, there are a few challenges in using CT and MRI techniques to reveal the extracranial facial nerve. Additionally, CT and MRI are unable to efficiently evaluate the facial nerve edema. Thus, to support the clinical diagnosis ultrasonography (US) should be combined with CT and MRI.

Prior to the advancement of imaging methods, electrophysiological methods were used to evaluate the outcomes in 
the treatment of facial nerve diseases, such as Bell's palsy (10). Electromyography, facial nerve conduction and blink reflexes were among the tests used for these evaluations (10). These methods had high sensitivity in the diagnosis of early neuritis, but their low specificity impeded the prediction of treatment effectiveness.

Since Fornage (11) reported on the sonographic evaluation of peripheral nerves two decades ago, high-frequency ultrasonography (HFUS) has evolved rapidly. HFUS is a useful tool for observing the facial nerves in patients with facial paralysis. In recent years, a variety of evaluation techniques and US devices have been reported (12). HFUS is a non-invasive method that is cost-effective and is capable of demonstrating the lesion structure together with the course of the affected nerve (13).

In the present study, US was employed to observe the normal and abnormal facial nerve. This method was compared to well-known electrophysiological techniques, such as facial nerve M-wave detection. The results showed that HFUS is able to provide a more convenient and effective method for diagnostic, prognostic and treatment evaluation purposes for patients suffering from Bell's palsy.

\section{Materials and methods}

General. Informed consent was obtained from the patients and their families. The present study was approved by the Ethics Committee of Capital Medical University (Beijing, China). A total of 104 healthy volunteers, 40 patients with acute onset of Bell's palsy and 30 patients who underwent three-month routine therapy for their disease were included in the present study. Healthy volunteers and patients underwent HFUS examination and VII nerve conduction.

All 104 healthy volunteers met the criteria for inclusion in the control group (CG) (mean age, 31; range, 15-57; 55 males). The experimental group A (EGA) included 40 patients (mean age, 28; range, 18-48; 28 males) with acute onset of unilateral Bell's palsy, with all the patients being examined within one week from onset of the symptoms. Each patient's facial nerve was measured clearly. Each patient was examined by an experienced neurologist who confirmed the diagnosis and classified the severity of the disease according to the Facial NGS2.0 grading system. Results from the examination of EGA classified the patients between grade III and VI (1). The experimental group A (EGB) included 30 patients (mean age, 30; range, 18-60; 15 males). All the patients in the EGB, who had undergone three-month of routine therapy, were evaluated and classified as grade I and II.

The exclusion criteria for this study included idiopathic facial nerve disease and peripheral nerve lesions caused by complications of diabetes, stroke, traumatic brain injury, otitis media or tumor. Additionally, prior to the electrophysiological examinations, the patients did not use any medication that potentially affected the results.

HFUS examination. HFUS examinations were conducted using a MHz linear-array probe (C12-5, Philips iU22, Philips, Amsterdam, The Netherlands) by an experienced operator. As the study progressed, every effort was made to scan volunteers in a completely supine position, allowing for better visualiza-

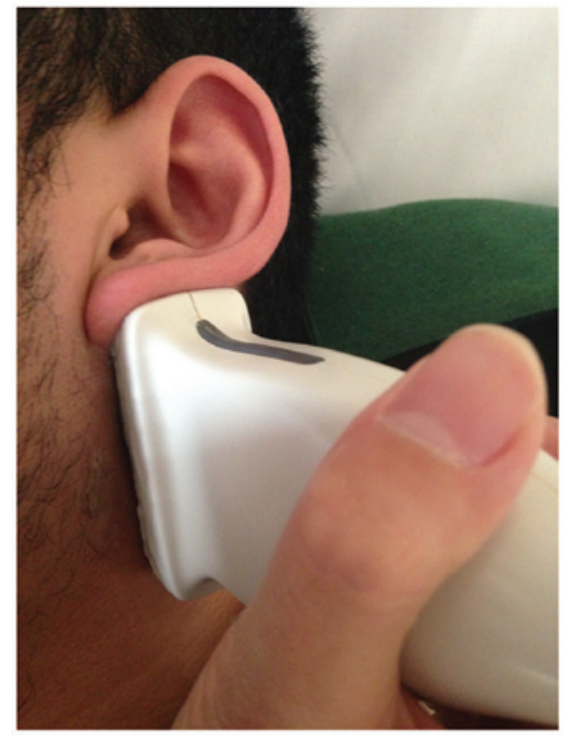

Figure 1. The method of high-frequency ultrasonography examination in longitudinal planes in a volunteer case.

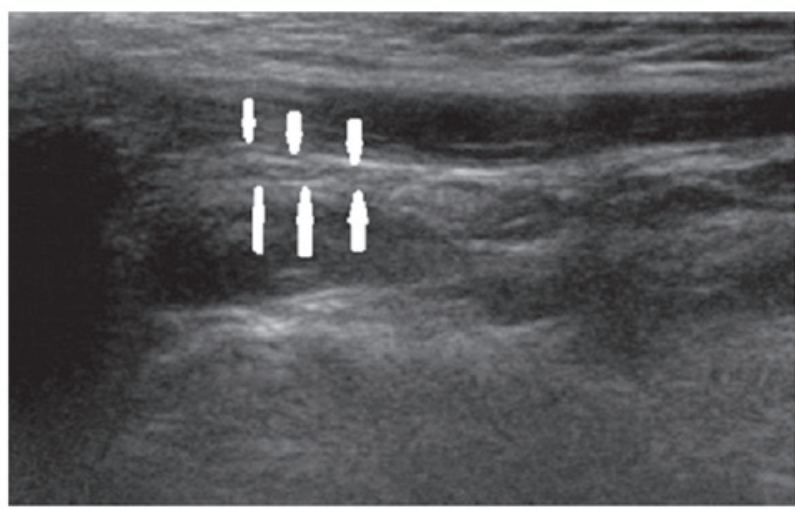

Figure 2. Longitudinal sonograms of the normal facial nerve.

tion on the small nerve structures. The facial nerve in the longitudinal view was identified at the mastoid region at the point from which it emerged from the stylomastoid foramen. At this site, it traversed anteriorly into the parotid gland substance prior to dividing into five branches (10). In the first step, the left end of the probe was aimed at the stylomastoid foramen, and subsequently, the probe was tilted outward and away from the parotid gland. The shallow hypoechoic muscle was revealed in the acoustic window. The region between the stylomastoid foramen exit and the parotid gland was visualized.

Fig. 1 shows the US examination method in longitudinal planes in a volunteer. The normal facial nerve in the longitudinal plane had a relatively hyperechoic sheath compared to the surrounding muscles, exhibiting a linear fascicular appearance with an oval hypoechoic structure and the spot echo surrounding the hyperechoic film strip in the transverse sonogram. By contrast, the abnormal facial nerve was often swollen with decreased echo, hyperechoic sheath and the fascicular pattern was obscure. Patients in EGA were scanned within the first week of the onset of their symptoms. Patients in EGB were scanned when they had a favorable outcome three months subsequent to clinical presentation. Figs. 2-4 show 


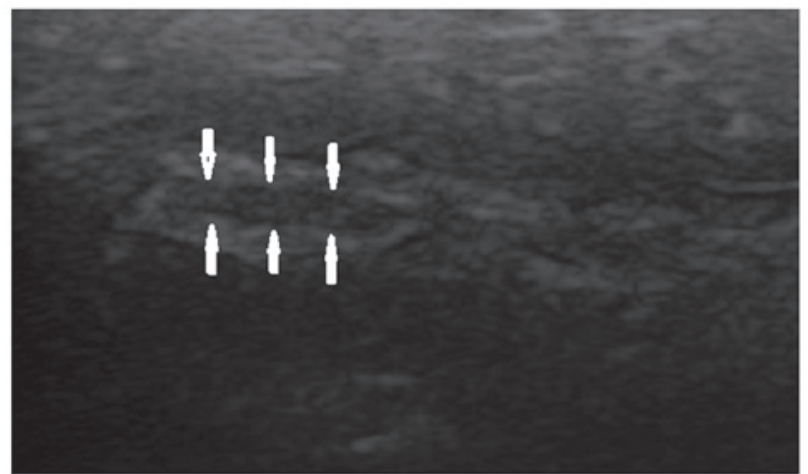

Figure 3. Longitudinal sonograms of the affected facial nerve after 1 week.

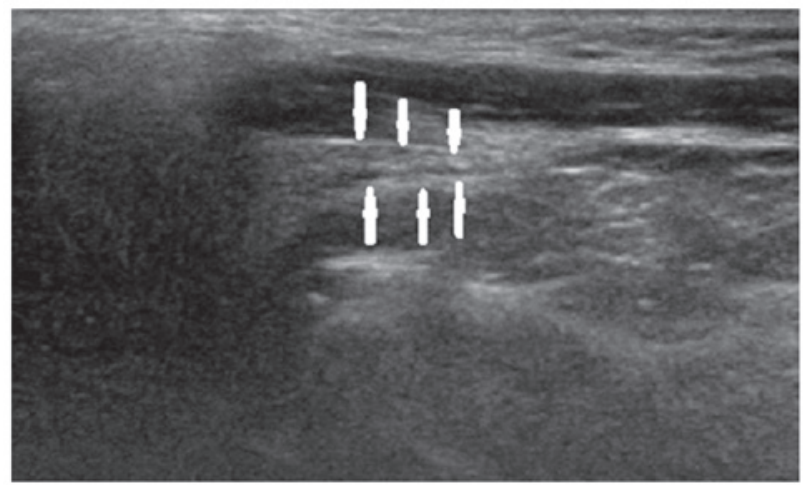

Figure 4. Longitudinal sonograms of the affected facial nerve after 3 months.

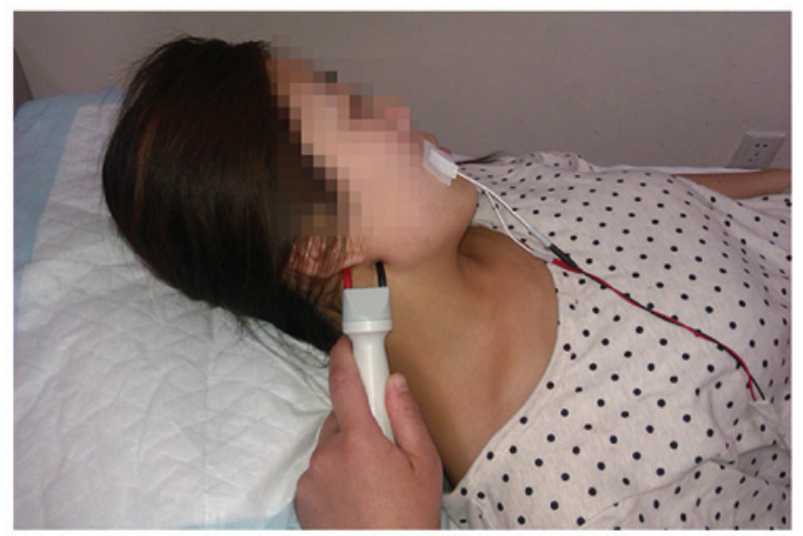

Figure 5. The method of nerve conduction for facial nerve in our volunteers.

longitudinal sonograms of the normal and affected facial nerve in 1 week and 3 months, respectively.

From the most proximal to the distal visualized portions, the average surface area and diameter of facial nerve crosssections was measured. The average length as well as the depth from the skin, visible length and the facial nerve to facial artery ratio of the same location were also measured.

Nerve conduction. Electromyograph and evoked potential measurement (Keypoint; Dantec Dynamics A/S, Kongeriget, Denmark) was performed following marking of the body surface according to US observation. Subsequently, the facial nerve M-wave detection was selected. Electrophysiological

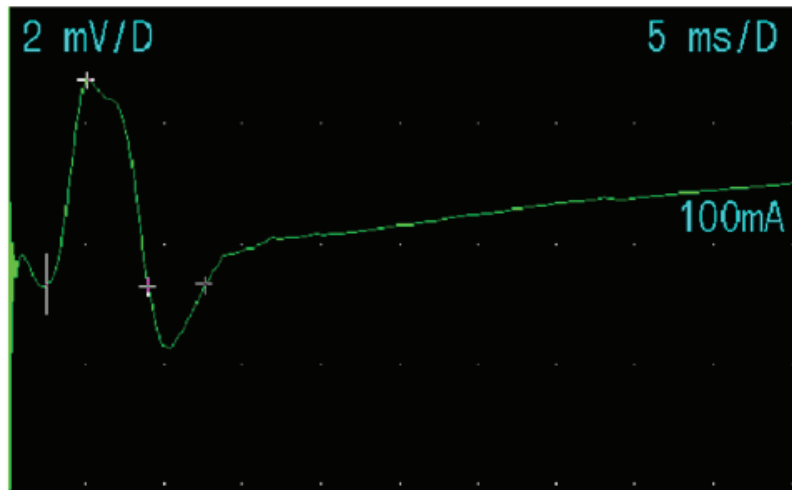

Figure 6. M-wave of the facial nerve in the normal facial nerve.

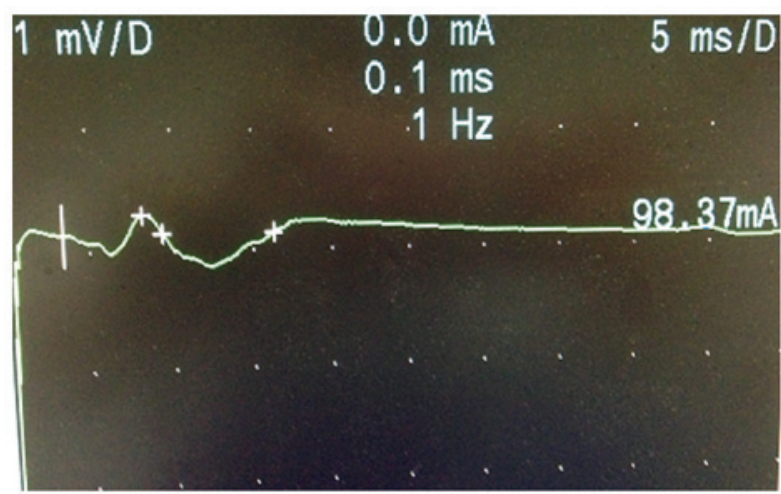

Figure 7. M-wave of the facial nerve in an abnormal subject.

detection was performed at room temperature $\left(20-30^{\circ} \mathrm{C}\right)$. Patients were in the supine position, with eyes closed. Surface electrodes were placed on the orbicularis oculi muscle and the orbicularis oris muscle. Reference electrodes were placed on the chin and the ground wire was placed on the arm. For patients with visible facial nerve at the surface, stimulation was applied. The sensitivity was $0.2 \mathrm{mV} / \mathrm{div}$ and the scanning speed was $5 \mathrm{~ms} / \mathrm{div}$. The initial intensity of the stimulus induced a maximum compound muscle action potential (CMAP), which was increased to achieve a stronger stimulation of $10-30 \%$. The stimulation time was $0.1 \mathrm{msec}$ and the analysis time was 20 msec. CMAP amplitude and distal motor latency of the facial nerve were observed. Fig. 5 shows the method for nerve conduction of the left facial nerve in the volunteers of the present study. Figs. 6 and 7 show the M-wave of the facial nerve in the normal and abnormal facial nerve subjects.

Statistical analysis. Data are presented as mean \pm standard deviation. The measurement data for the facial nerves were used for the paired t-test. The differences between groups were analyzed using one-way ANOVA and numerical data were analyzed using the $\chi^{2}$ test. The SPSS statistical software (SPSS, Inc., Chicago, IL, USA) package was used for the statistical analysis. $\mathrm{P}<0.05$ indicated statitistically significant differences.

\section{Results}

Results of the present study revealed that the mean normal facial nerve diameter was $0.16 \pm 0.03 \mathrm{~cm}$ and the cross-sectional 
Table I. HFUS and M-wave detection of the facial nerves about CG, EGA and EGB.

\begin{tabular}{|c|c|c|c|c|c|c|c|c|c|}
\hline \multirow[b]{2}{*}{ Characteristics } & \multicolumn{3}{|c|}{ CG } & \multicolumn{3}{|c|}{ EGA } & \multicolumn{3}{|c|}{ EGB } \\
\hline & Left & Right & $\mathrm{p}$-value & AS & US & $\mathrm{p}$-value & AS & US & P-value \\
\hline Diameter, cm & $0.16 \pm 0.02$ & $0.16 \pm 0.03$ & 0.027 & $0.20 \pm 0.02$ & $0.14 \pm 0.02$ & 0.000 & $0.19 \pm 0.03$ & $0.14 \pm 0.02$ & 0.000 \\
\hline $\mathrm{CSA}, \mathrm{cm}^{2}$ & $0.05 \pm 0.01$ & $0.05 \pm 0.01$ & 0.006 & $0.09 \pm 0.01$ & $0.04 \pm 0.01$ & 0.000 & $0.07 \pm 0.01$ & $0.04 \pm 0.01$ & 0.000 \\
\hline Depth, cm & $0.86 \pm 0.02$ & $0.84 \pm 0.13$ & NS & $0.85 \pm 0.02$ & $0.82 \pm 0.03$ & NS & $0.83 \pm 0.03$ & $0.84 \pm 0.12$ & NS \\
\hline $\mathrm{VL}, \mathrm{cm}$ & $0.99 \pm 0.14$ & $1.01 \pm 0.12$ & NS & $1.02 \pm 0.10$ & $1.11 \pm 0.12$ & NS & $1.10 \pm 0.08$ & $1.10 \pm 0.07$ & NS \\
\hline Hyperechioc & 97 & 96 & NS & 4 & 38 & 0.000 & 27 & 29 & NS \\
\hline Hypoechioc & 1 & 2 & NS & 36 & 2 & & 3 & 1 & \\
\hline Clear & 96 & 96 & NS & 10 & 36 & 0.000 & 16 & 28 & 0.000 \\
\hline Obscure & 2 & 2 & NS & 30 & 4 & & 14 & 2 & \\
\hline Delitescence, msec & $3.13 \pm 0.06$ & $2.94 \pm 0.06$ & NS & $3.66 \pm 0.66$ & $2.84 \pm 0.06$ & 0.000 & $2.94 \pm 0.38$ & $3.03 \pm 0.28$ & NS \\
\hline Amplitude, mV & $2.25 \pm 0.11$ & $2.24 \pm 0.12$ & NS & $1.43 \pm 0.75$ & $2.35 \pm 0.22$ & 0.000 & $2.22 \pm 0.89$ & $2.40 \pm 0.26$ & NS \\
\hline Length, $\mathrm{cm}$ & $11.1 \pm 1.21$ & $10.0 \pm 1.86$ & NS & $12.4 \pm 1.32$ & $10.4 \pm 1.24$ & NS & $12.5 \pm 1.02$ & $11.4 \pm 1.24$ & NS \\
\hline
\end{tabular}

HFUS, high-frequency ultrasonography; CG, control group; EGA, experimental group A; AS, affected side; US, uninjured side; CSA, cross-section area; Depth, from the skin to the facial nerve; VL, visual length; NS, not significant; Length, from measure point to mouth corner. Data are presented as mean \pm standard deviation.

Table II. Comparison of P-values between CG, EGA and EGB.

\begin{tabular}{lcccc}
\hline Characteristics & CG with EGA & CG with EGB & EGA with EGB & CG, EGA and EGB \\
\hline Diameter & 0.000 & 0.000 & 0.026 & 0.000 \\
Definition & 0.000 & 0.000 & 0.015 & 0.000 \\
Echogenicity & 0.000 & NS & 0.000 & 0.000 \\
Delitescence & 0.011 & NS & 0.000 & 0.012 \\
Amplitude & 0.028 & NS & 0.000 & 0.023 \\
\hline
\end{tabular}

CG; control group; EGA, experimental group A; NS, not significant.

area (CSA) was $0.05 \pm 0.01 \mathrm{~cm}^{2}$. The visual length and depth from the skin were $1.01 \pm 0.12$ and $0.85 \pm 0.13 \mathrm{~cm}$, respectively. The ratio (facial nerve to facial artery ratio) of the normal average right facial nerve was $1.06 \pm 0.27$.

Table I summarizes results obtained from all the patients. Some results of two sides of HFUS and M-wave in each group were significantly different. The CSA of the facial nerve was not perfectly round. The CSA was not calculated using the usual formula, but individual tracings were obtained to calculate the area using integration. The CSA of the facial nerve was drawn and the automatic measurement.

We found significant differences in nerve diameters in different groups $(\mathrm{P}<0.05)$. We also found significant differences in CSA, definition, echogenicity, delitescence and amplitude $(\mathrm{P}<0.05)$. The nerve diameters in the two experimental groups were greater than the $\mathrm{CG}$. Additionally, nerve diameters in EGA were larger than those in EGB. These results suggested that facial nerve edema improved following treatment (Table II).

In the EGA group, no significant correlation was identified for the severity grading nerve, diameter, CSA, delitescence and amplitude. A statistically significant correlation was observed for severity grading in the EGB during HFUS examinations, but the delitescence and amplitude had no statistical significance (Tables III and IV). The echogenicity of the nerve was restored, although the diameter and the definition of the nerve remained different in the EGB. The diameter and the definition of patients with severity grade II were different compared to grade I. Furthermore, there was no significant correlation among abnormal US diameter, delitescence and amplitude.

\section{Discussion}

HFUS examination in normal and abnormal nerves. The HFUS method has proved its efficacy over a wide range of nerve types as well as a wide range of ages and respective body sizes (13). The current approach for localizing and assessing the severity of nerve injuries involves accurate clinical history, physical examination and electrodiagnostic studies (14). However, such diagnostic tests do not reveal the exact location or the cause of the lesions. Additionally, they do not provide spatial information concerning nerves and surrounding structures. High-resolution US offers several advantages over other existing techniques. HFUS is faster, has superior spatial 
Table III. Comparison of severity grading for EGA at HFUS and M-wave detection.

\begin{tabular}{lcccc}
\hline Grade & III & IV & V-VI & P-value \\
\hline Diameter, mm & $0.21 \pm 0.02$ & $0.20 \pm 0.04$ & $0.21 \pm 0.02$ & NS \\
Definition, clear/obscure & $8 / 2$ & $4 / 4$ & $4 / 18$ & NS \\
Echogenicity, hyper/hypo & $6 / 4$ & $3 / 5$ & $2 / 20$ & NS \\
Delitescence, msec & $3.78 \pm 0.64$ & $3.75 \pm 0.43$ & $3.57 \pm 0.74$ & NS \\
Amplitude, mV & $1.01 \pm 0.18$ & $1.09 \pm 0.22$ & $1.75 \pm 0.87$ & NS \\
Number & 10 & 8 & 22 &
\end{tabular}

HFUS, high-frequency ultrasonography; EGA, experimental group A; NS, not significant. Data are provided as mean \pm standard deviation.

Table IV. Comparison of severity grading for EGB at HFUS and $\mathrm{M}$-wave detection.

\begin{tabular}{lccc}
\hline Grade & I & II & p-value \\
\hline Diameter, mm & $0.16 \pm 0.02$ & $0.21 \pm 0.02$ & 0.000 \\
Definition, clear/obscure & $8 / 3$ & $8 / 11$ & 0.04 \\
Echogenicity, hyper/hypo & $10 / 1$ & $17 / 2$ & $\mathrm{NS}$ \\
Delitescence, msec & $2.86 \pm 0.43$ & $2.98 \pm 0.36$ & $\mathrm{NS}$ \\
Amplitude, mV & $2.07 \pm 0.70$ & $2.14 \pm 0.92$ & $\mathrm{NS}$ \\
Number & 11 & 19 &
\end{tabular}

HFUS, high-frequency ultrasonography; EGB, experimental group B; NS, not significant. Data are provided as mean \pm standard deviation.

resolution and is more dynamic, therefore it is an optimal option for patients (15). A clear understanding of neural anatomy is crucial in designing a successful clinical therapy. Appropriate treatment can be designed and planned only when there is a clear image with regard to the function and distribution of nerve bundles and the anatomical relationship with the adjacent structures. For surgeons, identification of the location and the tract of the facial nerve anatomy is imperative.

For ultrasound radiologists, detection of the correct images of facial nerve and avoidance of the interferences from tendons, muscles and parotid gland is important. US examinations are operator-dependent and require experience in superficial soft-tissue structures, which have a relatively long learning curve. The present study examined the feasibility of performing measurements on normal and abnormal facial nerves by ultrasound radiologists without facial nerve expertise. Parameters such as diameter, CSA, depth from the skin and visible length were used as references in the absence of nerve electrophysiological cases. Stylomastoid foramen is a significant characteristic for identifying facial nerves. Tendons and nerves are difficult to identify as they are linked together. Thus, technicians should move the probe gently to identify the facial nerve entering the parotid gland. Kele (16) reported that in individuals in good physical condition, cranial nerves, such as the vagal and accessory nerves, can be visualized regularly. The nerves have cable-like structures and appear on the transversal sections as round to oval hyperechoic structures. They are surrounded by an echogenic rim representing the epifascicular epineurium and the perineurial fatty tissue. The sonographic echo pattern (texture) is known as 'honeycomb-shaped' (17). As mentioned earlier, Hagino et al (3) in his study established that facial paralysis patients manifested facial nerve edema in only in few (15) cases of mastoidectomy and facial nerve canal operation.

In the present study, we found significant differences between the two sides of normal nerve diameters. In several volunteers along with the age, even with the exclusion of idiopathic facial nerve disease and peripheral nerve lesions caused by complications from diabetes, stroke, traumatic brain injury, otitis media or tumor, patients inevitably encounter cavities, periodontitis and other oral diseases or even issues with chewing. These issues may be detected in electrophysiology or have a direct effect on the nerve itself. However, electrophysiology does not detect lesions that are evident with HFUS. Nevertheless, the differences between the two methods were $<0.02 \mathrm{~cm}$, indicating that there was no clinical significance.

HFUS identifies patients with grading II. We also analyzed Bell's palsy according to Facial Nerve Grading. The patients were generally classified as grade III and above; therefore, we assigned grades III-VI for the patients in the EGA. The patients were treated actively and continued to be treated when clinical symptoms persisted three months following the initial therapy. Patients considered the disease cured following amelioration of grade from VI to I or II. In these cases, it was necessary to judge objectively whether to continue patient treatment. Evaluation of the curative effects for grade I and II patients had more clinical significance. There was no statistical significance in neural function detection in patients as grade I or II. The US findings are clinically important as they can provide accurate guidance to patients and physicians with regard to whether to continue the treatment for facial paralysis.

The nerve diameters in the two experimental groups were greater than the CG. In the EGA, nerve diameters were larger than those in the EGB. These results suggested that facial nerve edema had the tendency to improve following treatment. The echogenicity of the nerve was restored, although there were differences in the diameters and definitions of nerve in the EGB. The diameter and definition of patients with grade II severity were different to those with grade I. Consequently, the patients with grade II were judged to be eligible to continue their treatment. 
A previous report revealed that the measurement of facial nerve diameter was an appropriate predictor for good prognosis (with positive predictive value of $100 \%$ ). However, it was not a useful predictor in the case of an unsatisfactory prognosis (negative predictive value of $77 \%$ ). HFUS was highly correlated with clinical grade outcomes (18). Our results are important because we examined the diameter and CSA, and analyzed the characteristics of facial nerve images, such as definition and echogenicity. The results from the current study also confirmed that US detection correlated well with House-Brackemann Facial Nerve Grading.

US combined with electrophysiological and MRI support clinical diagnosis. The neural electrophysiological verification of HFUS measurement of the facial nerve can effectively confirm the results obtained by ultrasound and thus ensure its accuracy. We selected the M-wave of nerve electrophysiology because the operation was simple and the observation results were obvious (19). In comparison to M-wave between CG, EGA and EGB, the delitescence and amplitude were statistically significant for severity grades III to VI in the EGA. Thus, the results of $\mathrm{M}$-wave determined facial paralysis with high sensitivity and were closely associated with clinical symptoms. We found no statistical significance for grading in the EGA and EGB, suggesting that US was a superior technique compared to electrophysiological studies in outcome prediction.

MRI scans may be used for imaging purposes for the facial nerve from the brainstem to the fundus of the internal auditory canal (8). MRI normally visualizes soft tissues well and is better suited for intracranial lesions. MRI may actually reveal lesions and vascular rather than structural changes visible with US (3). US combined with CT and MRI may support clinical diagnosis.

Limitations. Limitations were found in evaluating peripheral nerves. US examinations were operator-dependent and required experience in the superficial soft-tissue structures that had a relatively long learning curve. The number of cases in the EGA and EGB were limited. M-wave was only one of the numerous electrophysiological techniques and we did not employ other techniques, such as blink reflex and F-wave.

In conclusion, HFUS as an adjunct to neural electrophysiology is highly useful in the establishment of the normal values of facial nerve. HFUS is also useful in the process of evaluation and prognosis of Bell's palsy. HFUS confirmed that facial nerve edema improved following treatment and was useful in the determination of whether patients with NGS2.0 grading II required additional treatment. HFUS also proved itself to be superior to electrophysiological studies in outcome prediction.

\section{References}

1. Gupta S, Mends F, Hagiwara M, Fatterpekar G and Roehm PC: Imaging the facial nerve: a contemporary review. Radiol Res Pract 2013: 248039, 2013.

2. Vrabec JT, Backous DD, Djalilian HR, Gidley PW, Leonetti JP, Marzo SJ, Morrison D, Ng M, Ramsey MJ and Schaitkin BM; Facial Nerve Disorders Committee: Facial Nerve Grading System 2.0. Otolaryngol Head Neck Surg 140: 445-450, 2009.

3. Hagino K, Tsunoda A, Tsunoda R and Kishimoto S: Measurement of the facial nerve caliber in facial palsy: implications for facial nerve decompression. Otol Neurotol 32: 686-689, 2011.

4. Saatçi I, Sahintürk F, Sennaroğlu L, Boyvat F, Gürsel B and Besim A: MRI of the facial nerve in idiopathic facial palsy. Eur Radiol 6: 631-636, 1996.

5. Kress B, Griesbeck F, Stippich C, Bähren W and Sartor K: Quantitative analysis of MRI intensity in of the major petrosal nerve in patients with idiopathic facial paralysis. Nervenarzt 75 : 124-127, 2004 (In German).

6. Kinoshita T, Ishii K, Okitsu T, Okudera T and Ogawa T: Facial nerve palsy: evaluation by contrast-enhanced MR imaging. Clin Radiol 56: 926-932, 2001

7. Nakata S, Mizuno T, Naganawa S, Sugiura M, Yoshida T, Teranishi M, Sone M and Nakashima T: 3D-FLAIR MRI in facial nerve paralysis with and without audio-vestibular disorder. Acta Otolaryngol 130: 632-636, 2010.

8. Murphy TP: MRI of the facial nerve during paralysis. Otolaryngol Head Neck Surg 104: 47-51, 1991.

9. Mu X, Quan Y, Shao J, Li J, Wang H and Gong R: Enlarged geniculate ganglion fossa: CT sign of facial nerve canal fracture. Acad Radiol 19: 971-976, 2012.

10. Sittel C and Stennert E: Prognostic value of electromyography in acute peripheral facial nerve palsy. Otol Neurotol 22: 100-104, 2001.

11. Fornage BD: Peripheral nerves of the extremities: imaging with US. Radiology 167: 179-182, 1988.

12. Vakharia KT, Henstrom D, Lindsay R, Cunnane MB, Cheney M and Hadlock T: Color Doppler ultrasound: Effective monitoring of the buried free flap in facial reanimation. Otolaryngol Head Neck Surg 146: 372-376, 2012.

13. Vlad V and Iagnocco A: Ultrasound of the knee in rheumatology. Med Ultrason 14: 318-325, 2012.

14. Yildirim AO, Oken OF, Unal VS, Esmer AF, Gülçek M and Uçaner A: Avoiding iatrogenic radial nerve injury during humeral fracture surgery: a modified approach to the distal humerus. Acta Orthop Traumatol Turc 46: 8-12, 2012.

15. Karabay N, Toros T, Ademoğlu Y and Ada S: Ultrasonographic evaluation of the iatrogenic peripheral nerve injuries in upper extremity. Eur J Radiol 73: 234-240, 2010.

16. Kele H: Ultrasonography of peripheral nerves system. New Trends in Neurosonology and Cerebral Hemodynamics-an Update 1: 417-421, 2012.

17. Suk JI, Walker FO and Cartwright MS: Ultrasonography of peripheral nerves. Curr Neurol Neurosci Rep 13: 328, 2013.

18. Lo YL, Fook-Chong S, Leoh TH, Dan YF, Lee MP, Gan HY and Chan LL: High-resolution ultrasound in the evaluation and prognosis of Bell's palsy. Eur J Neurol 17: 885-889, 2010.

19. Ishikawa M, Namiki J, Takase M, Kojima A and Kawase T: F-waves of the facial muscles in healthy control subjects and in patients with peripheral facial nerve disturbance. Electromyogr Clin Neurophysiol 39: 167-174, 1999.

\section{Acknowledgements}

This study was supported in part by the Department of Neurology and Traditional Chinese Medicine in Beijing Chaoyang Hospital, Capital Medical University (Beijing, China). 